\title{
A scoping review of pre-hospital technology to assist ambulance personnel with patient diagnosis or stratification during the emergency assessment of suspected stroke
}

Hannah A Lumley ${ }^{1 *}$, Darren Flynn², Lisa Shaw ${ }^{1}$, Graham McClelland ${ }^{1,3}$, Gary A Ford ${ }^{4}$, Phil M White ${ }^{1,5}$ and Christopher I Price ${ }^{1,6}$

\begin{abstract}
Background: Pre-hospital identification of key subgroups within the suspected stroke population could reduce delays to emergency treatment. We aimed to identify and describe technology with existing proof of concept for diagnosis or stratification of patients in the pre-hospital setting.

Methods: A systematic electronic search of published literature (from 01/01/2000 to 06/06/2019) was conducted in five bibliographic databases. Two reviewers independently assessed eligibility of studies or study protocols describing diagnostic/stratification tests (portable imaging/biomarkers) or technology facilitating diagnosis/ stratification (telemedicine) used by ambulance personnel during the assessment of suspected stroke. Eligible descriptions required use of tests or technology during the actual assessment of suspected stroke to provide information directly to ambulance personnel in the pre-hospital setting. Due to study, intervention and setting heterogeneity there was no attempt at meta-analysis.

(Continued on next page)
\end{abstract}

\footnotetext{
* Correspondence: Hannah.lumley@newcastle.ac.uk

${ }^{1}$ Population Health Sciences Institute, Faculty of Medical Sciences, Newcastle University, Newcastle upon Tyne, UK

Full list of author information is available at the end of the article
}

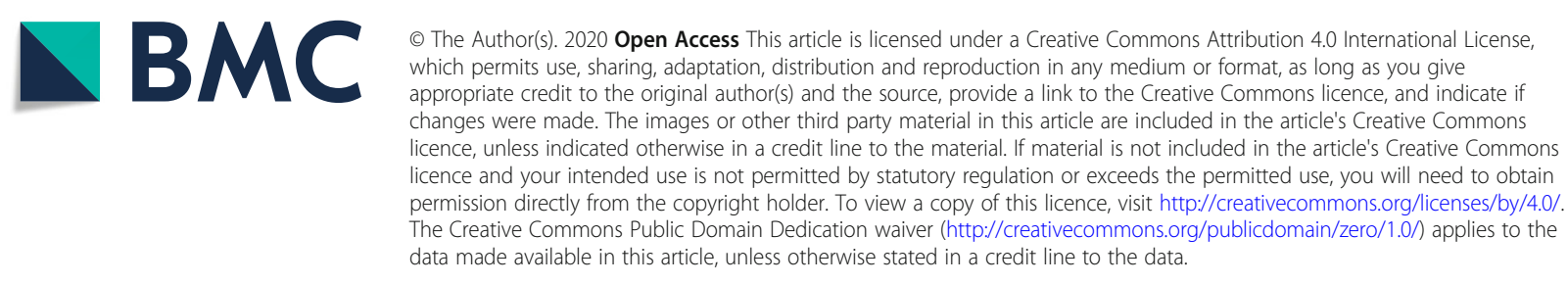


(Continued from previous page)

Results: 2887 articles were screened for eligibility, 19 of which were retained. Blood biomarker studies ( $n=2)$ were protocols of prospective diagnostic accuracy studies, one examining purines and the other a panel of known and novel biomarkers for identifying stroke sub-types (versus mimic). No data were yet available on diagnostic accuracy or patient health outcomes. Portable imaging studies $(n=2)$ reported that an infrared screening device for detecting haemorrhages yielded moderate sensitivity and poor specificity in a small study, whilst a dry-EEG study to detect large vessel occlusion in ischaemic stroke has not yet reported results. Fifteen evaluations of pre-hospital telemedicine were identified (12 observational and 3 controlled comparisons) which all involved transmission of stroke assessment data from the pre-hospital setting to the hospital. Diagnosis was generally comparable with hospital diagnosis and most telemedicine systems reduced time-to-treatment; however, it is unknown whether this time saving translated into more favourable clinical outcomes. Telemedicine systems were deemed acceptable by clinicians.

Conclusions: Pre-hospital technologies to identify clinically important subgroups amongst the suspected stroke population are in development but insufficient evidence precludes recommendations about routine use in the prehospital setting. Multi-centre diagnostic accuracy studies and clinical utility trials combining promising technologies are warranted.

Keywords: Stroke, Pre-hospital, Stratification, Diagnosis, Technology, Biomarkers, Imaging, Telemedicine, Ambulance, Paramedic

\section{Introduction}

Stroke is a medical emergency responsible for a high global burden of mortality and disability, but the outlook is improved by rapid treatment of specific subgroups; such as intravenous thrombolysis for selected ischaemic stroke presentations [1, 2] and mechanical thrombectomy (MT) for large vessel occlusion (LVO) [3]. Due to the increasing centralisation of acute stroke care at specialist facilities [4], early identification and stratification is needed to ensure prompt arrival at the correct hospital for efficient treatment.

For most individuals with acute stroke, the first healthcare contact is a paramedic. However, accurate identification is challenging in the pre-hospital setting due to heterogeneous clinical presentations, time pressure and an absence of simple diagnostic technology. Currently, ambulance personnel use symptom checklists, such as the Face Arm Speech Test (FAST) [5], which have moderate-to-good sensitivity but lower levels of specificity, such that $30-50 \%$ of suspected stroke patients later receive an alternative 'mimic' diagnosis [5-7]. Once in hospital, stroke and the aetiological sub-type are confirmed via specialist review and brain imaging (computed tomography (CT) and/or magnetic resonance imaging (MRI)) so that mimic cases are excluded and appropriate treatments can be given. In some settings this assessment has been taken to patients using highly equipped 'mobile stroke unit' ambulances, with evidence that thrombolysis can be delivered more rapidly $[8,9]$. However, current models require a stroke specialist to be present in the vehicle which is not the standard approach for emergency care provision in many healthcare systems.
Currently, key stroke subgroups cannot be identified until after hospital arrival. There is no clinical assessment process which can accurately differentiate between ischaemic and haemorrhagic stroke, and mimics. Now there is overwhelming evidence favouring MT treatment for patients with more severe stroke due to LVO, this group (approximately 10\% [10]) should be found as early as possible for direction to those centres able to provide treatment. Symptom severity scores have been developed to assist pre-hospital LVO recognition, but primary evidence of impact on outcome is lacking and accuracy is reduced by mimic, haemorrhagic and non-LVO presentations exhibiting severe symptoms [11, 12].

Pre-hospital assessment based upon symptom checklists alone creates substantial inefficiencies for stroke patients (treatment delays), mimics (displacement to specialist units) and services (additional demands on resources). However, improvement in early stratification/ diagnosis of stroke and $\mathrm{LVO} / \mathrm{ICH}$ subgroups may be possible with emerging technologies which are deployable in standard ambulances [13]. Many are still at early stages of development and will require evaluation of feasibility and resource impact relative to their ability to differentiate, individually or in combination, between key subgroups during the first few hours after symptom onset [14].

\section{Aim}

The aim of this scoping review was to identify, categorise and report the capability of technologies where proof of concept exists for diagnosis and/or stratification of suspected stroke when used by ambulance personnel in the pre-hospital setting. 


\section{Objectives}

1. Describe and classify technologies intended to enhance pre-hospital diagnosis of stroke, and/or stratification of suspected stroke, based upon published studies and protocols.

2. Describe evidence for proof of concept and/or feasibility and/or accuracy of the technology according to its stage of development.

3. Describe the impact of the technology on patient care processes or outcomes if data are available.

\section{Review methods}

Methods using standard guidelines [15] have already been described in detail in an online protocol (CRD42018087611) [16].

\section{Search strategy}

Following exploratory searches, with reference to the scoping review question and in collaboration with an information scientist, a systematic search strategy combining MeSH/Web of Science categories and keywords/ topic searches was developed and applied to Medline, PubMed, Embase and Web of Science up to 06/06/2019. Hand searching of reference lists and citation searches of included studies were undertaken. Searches were also conducted in online trial registries to identify currently unpublished registered trials on candidate technologies not detected in the literature search. The search strategies are found in 'Additional file 1: Appendix 1'. Papers published before the year 2000 were excluded as there was little emphasis on stroke-specific assessment technologies prior to expansion of the evidence supporting intravenous thrombolysis.

Conference abstracts published after 2013 were identified by applying the search strategy to the databases, with searches limited to 'conference abstracts'. Grey literature was identified from contact with clinical prehospital care content experts. This was done to identify emerging technologies.

\section{Review criteria}

Primary quantitative, qualitative or mixed methods research studies and protocols, including feasibility and pilot studies, with abstracts published in English from any country were eligible for inclusion.

Eligible articles had to describe completed studies or protocols which met the following criteria:

1. (i) Direct diagnostic technologies (e.g. biomarkers) and/or (ii) adjunctive technology to facilitate stratification (e.g. telemedicine requiring equipment which isn't routinely present in standard ambulances).

2. Application to suspected stroke patients during clinical care.
3. Use by ambulance personnel including paramedics, emergency medical technicians or other clinicians routinely providing pre-hospital care in specific countries, e.g. EMS physicians in Germany $[17,18]$.

4. How the result would be available to EMS clinicians in the pre-hospital setting (prior to hospital admission).

5. Technology at any stage of development as long as it was (or protocols where it is planned to be) applied during ambulance care of suspected stroke.

Ambulance-based studies were excluded if the technology would not be transferrable to standard ambulances without them becoming specialised vehicles (e.g. Mobile Stroke Units which include Computed Tomography (CT) and a Point of Care lab). Reports which described paper-based algorithms, clinical rules and clinical scales, studies involving medical personnel not routinely present in the pre-hospital setting, hospitalbased studies, studies focused on paramedic training and studies which did not exclusively include populations with suspected stroke were also excluded.

\section{Study selection}

Two reviewers (HL and DF) independently assessed titles/abstracts (stage 1). The same two reviewers independently assessed full texts of retained studies for their conformity to the inclusion criteria using a study selection form. Disagreements at the second stage were resolved via discussion or adjudicated by a third reviewer (CP).

\section{Data extraction}

Data were independently extracted by two reviewers (HL and DF), with discrepancies solved via discussion or adjudication (CP). A data extraction form captured detailed information on studies and technologies (Additional file 1: Appendix 2). As no adequate framework to evaluate diagnostic technology studies existed for this purpose, a modified version of the TIDieR framework [19] was used to standardise descriptions. This included generic study information (e.g. authors; publication year, country, purpose [diagnostic and/or facilitation of care], research design and key findings) and detailed information about the technologies. As the scope of the review was worldwide, we did not use the European Union CE mark guidance on physical invasiveness, and a simpler classification system was used [20]: invasive (penetration/breaking of the skin or entry into a body cavity); minimally invasive (indirect observation of internal areas of the body); and non-invasive (no penetration or breaking of the skin). The stage of development was recorded as alpha (initial prototype stage), beta (later iteration of the prototype, feature complete but not finalised) or gamma (finalised product available for wider use) [21]. 


\section{Data synthesis}

As this was a scoping review, there was no a-priori plan for data meta-analysis. Extracted information was used to develop a taxonomy describing direct or facilitative stratification technologies according to their mode of action. Where reported, data on true/false positives and negatives were used to calculate sensitivity and specificity values. Studies were classified according to study design to inform recommendations based upon strength of evidence.

\section{Results}

A total of 4870 (2886 after deduplication) studies were identified via the search strategy (Fig. 1). One study was identified via citation searches. 94 potentially eligible articles were retained following initial screening. After obtaining and reviewing full texts (or abstracts where full texts were not available), 75 studies were excluded with reasons detailed in Fig. 1. In total, 19 studies were included, 8 of which were full text articles, 7 conference abstracts and 4 published protocols.

\section{Description of included studies}

Technologies employed by included studies [22-40] were best described using three categories: blood biomarkers [22, 23]; pre-hospital imaging [24, 25] and mobile telemedicine/telestroke (including transfer of audio and/or visual information or relevant data) [26-40]. These are summarised in Table 1.

Most studies were conducted in the USA [24, 26, 27, 34-39], followed by Germany [28-30] and Belgium [31$33]$. One study was conducted in each of the following countries: UK [23], Finland [22], Netherlands [25] and Sweden [40].

\section{Blood biomarkers}

We identified protocols for two currently active, large pre-hospital studies of blood biomarker technologies which are both prospective diagnostic accuracy designs [22, 23], summarised in Table 2.

The Helsinki Ultra-acute Stroke Biomarker Study [22] is an early stage, single-centre study aiming to establish diagnostic and predictive biomarkers for potential IVT candidates. The specific aims are to (i) identify ischemic stroke, transient ischemic attack, intracerebral haemorrhage and stroke mimics; (ii) identify patients not responding to IVT; (iii) identify patients with increased chance of IVT-related complications; and (iv) predict 90-day patient health outcomes using the modified Rankin Scale (mRS). The study will evaluate known stroke biomarkers, e.g. Glial Fibrillary Acidic Protein (GFAP) and NR2 peptide, and explore novel markers (during a discovery phase) via blood samples taken by EMS clinicians during transit. This study has yet to report on

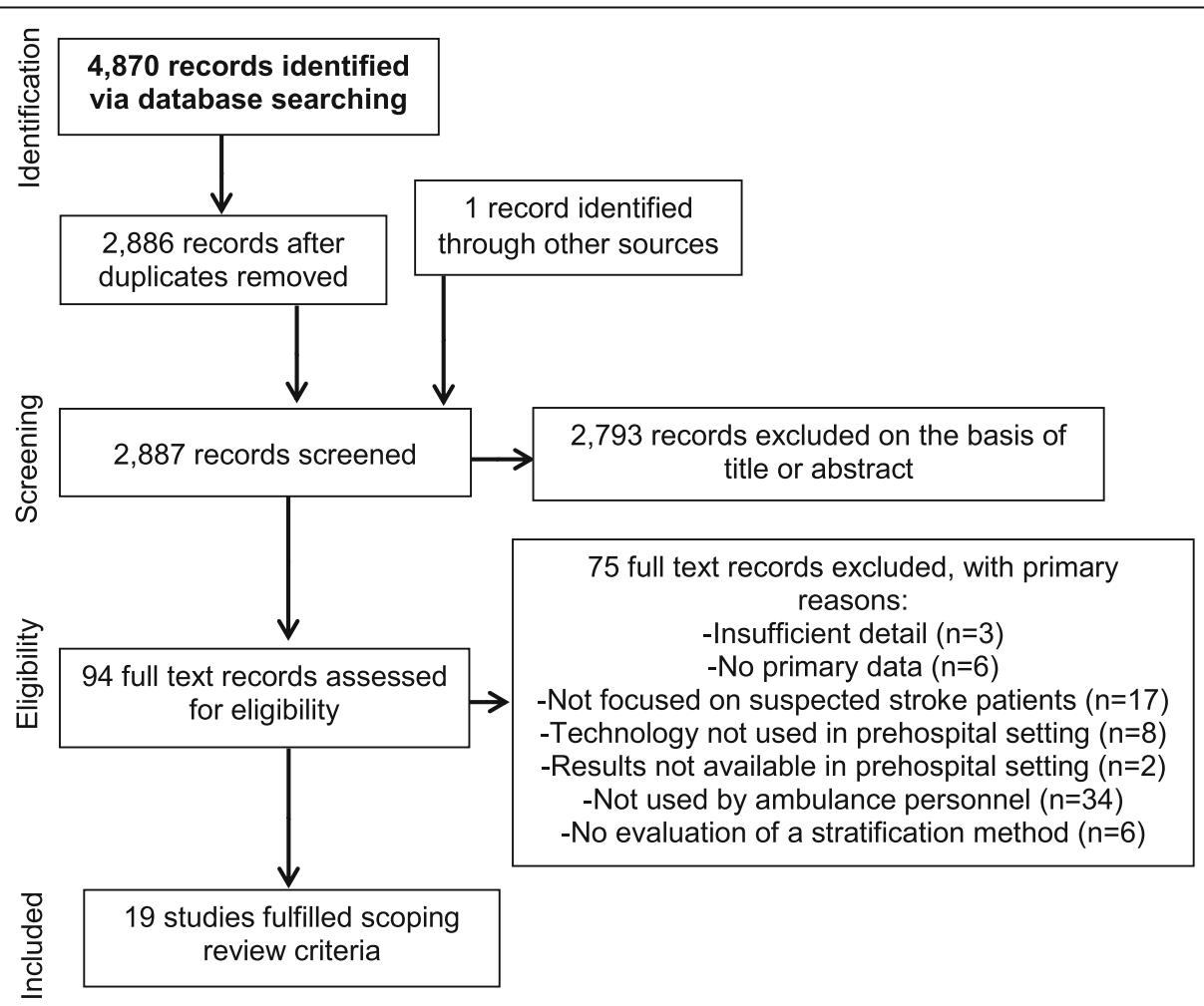

Fig. 1 Flow diagram summarising the process used to identify studies 


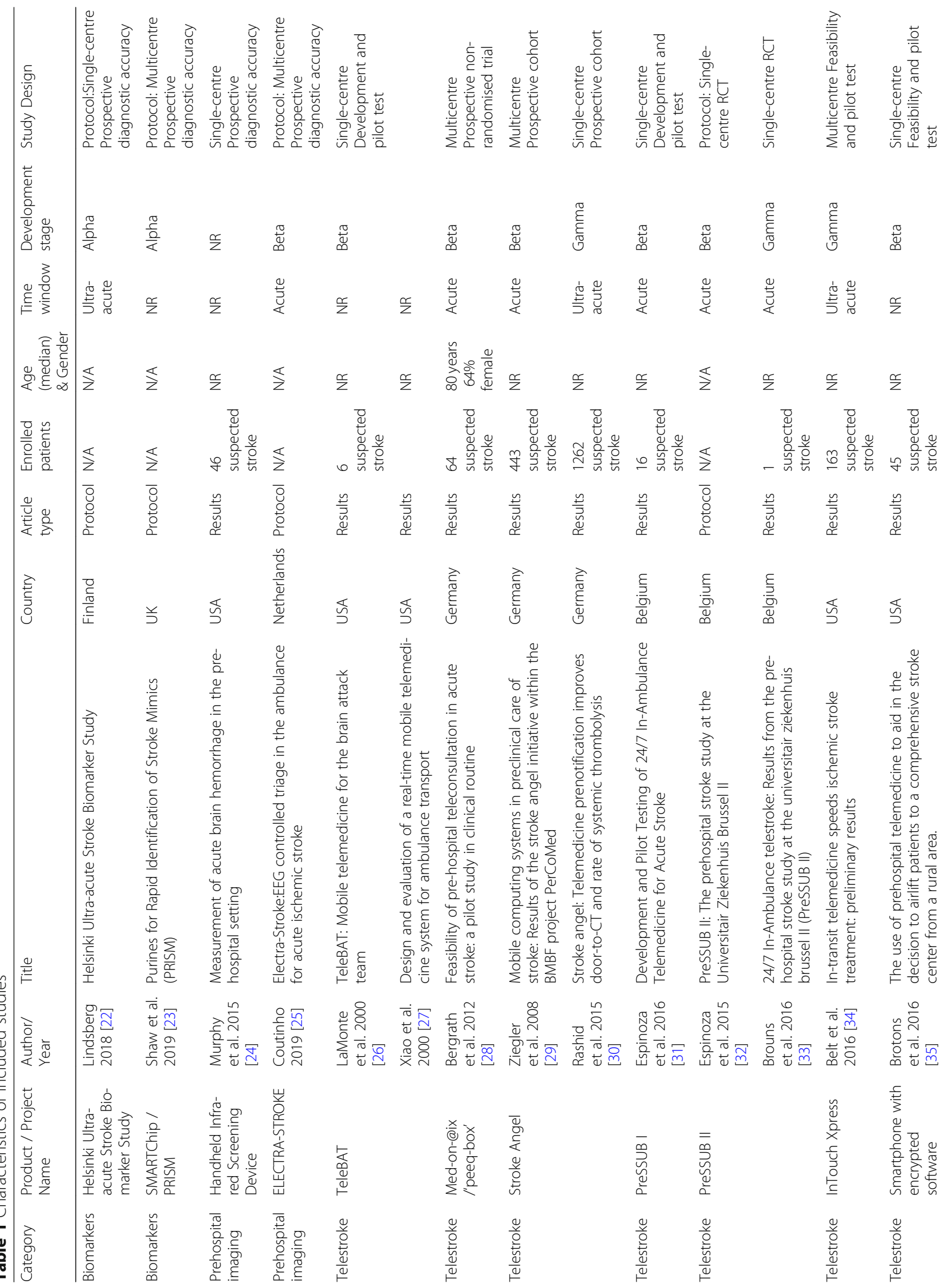




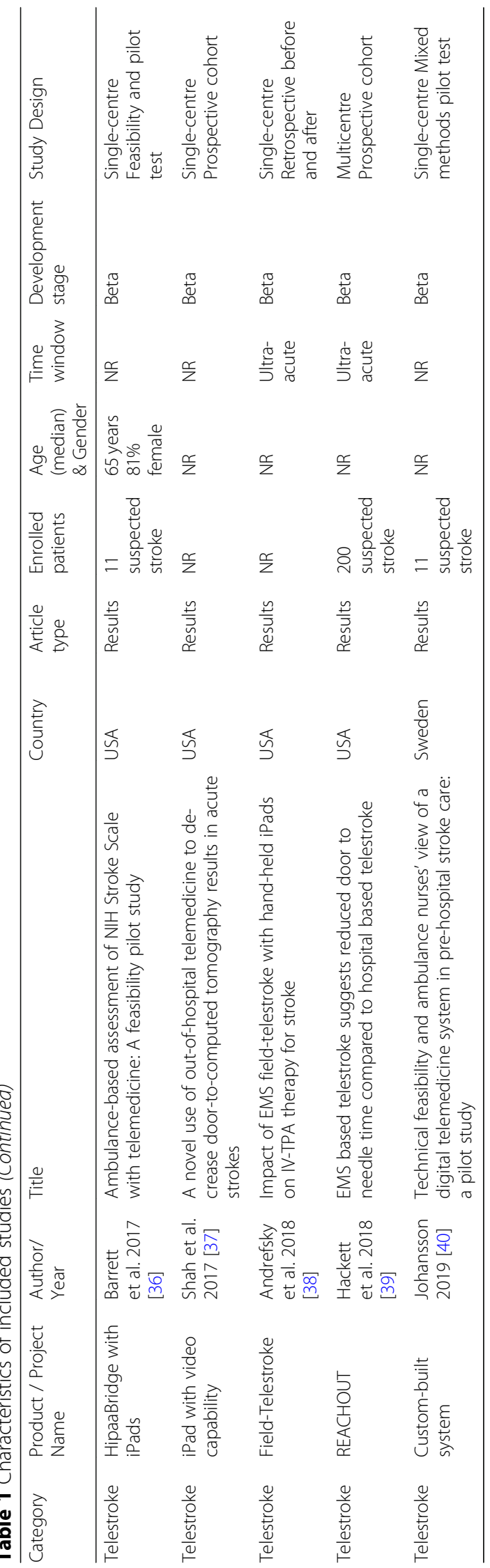




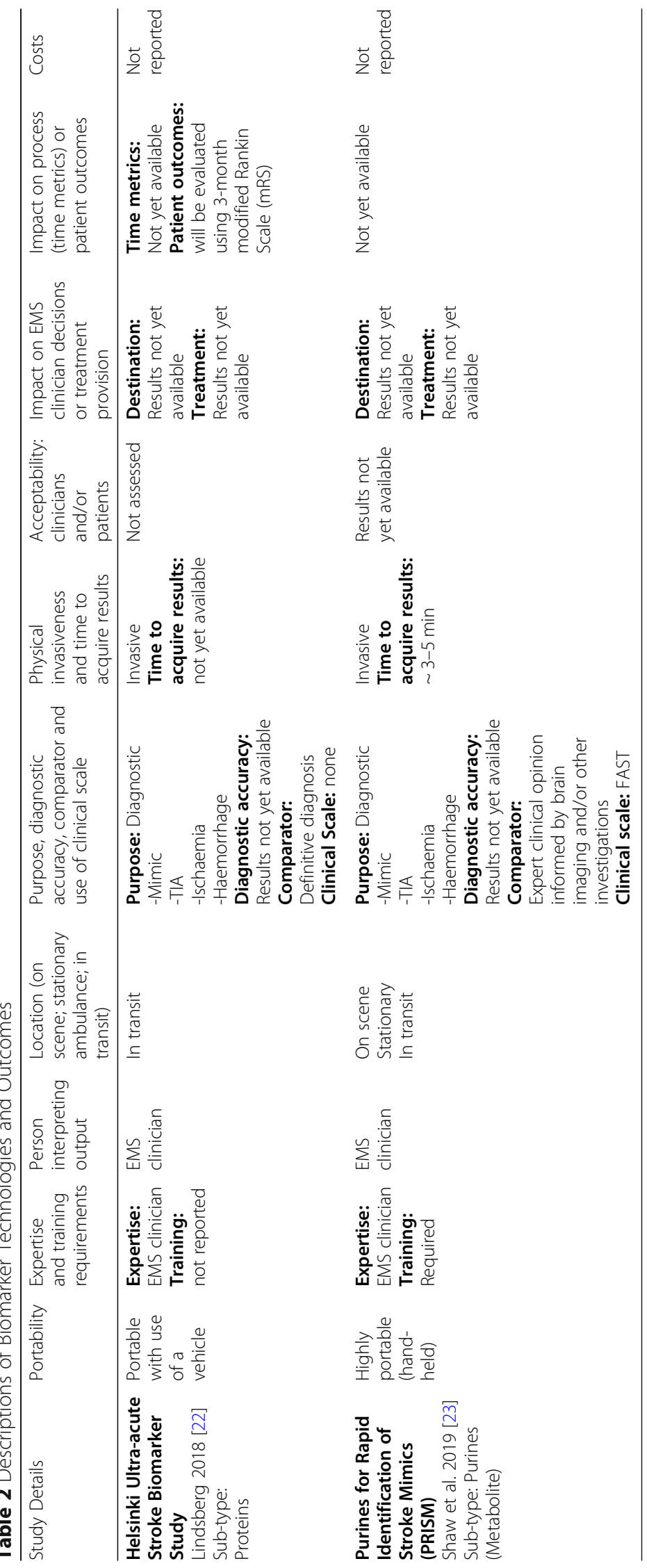


primary outcomes, although a precursor study [41] has reported the feasibility of implementing pre-hospital EMS biomarker sampling using a cannula adapter technique. It is not clear how EMS clinicians would obtain a result on scene in future (e.g. a point of care assay system) even if the panel is of value.

Purines for Rapid Identification of Stroke Mimics (PRISM) [23] is an early stage, multi-centre study aiming to assess the diagnostic accuracy of whole blood purine concentration using capillary blood sampling performed by trained EMS clinicians within $4 \mathrm{~h}$ of stroke symptom onset. The goal is to differentiate between stroke and stroke mimics, with a hospital sub-study investigating LVO. The point-of-care SMARTChip system, a handheld reader and disposable biosensor, developed by Sarissa Biomedical Ltd. in the UK, measures purines in a finger-prick blood sample. Purines are a by-product of cellular metabolism which accumulate rapidly during hypoxia (as occurs in stroke) and can be reliably detected in systemic arterial blood [42]. Results can be obtained within 3-5 min and paramedics require training to use this technology [43]. There is currently no published diagnostic accuracy or patient outcome data, however the technology is at a late stage of development which would facilitate deployment if of value. Neither biomarker protocol commented on the potential cost of the technologies.

\section{Pre-hospital imaging}

We identified two studies of non-invasive pre-hospital imaging technology $[24,25]$. These are summarised in Table 3. EEG is included here as 'imaging' since the intention is to produce information which correlates anatomically with cerebral tissue injury. From a study design perspective, these are prospective diagnostic accuracy studies.

Our review identified one small, single-centre study reporting on use of a handheld Infrared screening device in the pre-hospital setting in the USA by device-trained EMS clinicians during transit [24]. The stage of development was not reported. The purpose of the device was to discern between stroke types by detecting changes in blood flow. The authors found evidence that contralesional increases in blood flow indicate LVO. The device was compared with hospital-based CT on its ability to detect haemorrhagic stroke in 46 suspected stroke patients; 7 of these 46 patients had CT-confirmed haemorrhagic stroke. True and false positive/negative results are shown in Table 3; diagnostic accuracy was extrapolated from these figures. For haemorrhage versus mimic, sensitivity and specificity within the study population were 71 and $40 \%$ respectively. Sensitivity and specificity for haemorrhage versus ischaemia were 71 and $43 \%$ respectively. Although all haemorrhagic strokes were identified by the device, the poor specificity may limit clinical use. Paramedics considered the scan too long for stroke patients and consequent speeding up of the patient scan time increased false positives. Faster ambulance speed also increased false positive rates, and the device was difficult to use reliably with patients unable to lie still. There was no impact on destination decisions or reported change in patient outcomes.

'Dry' electrode cap Electroencephalography (EEG) will be used in the ELECTRA-STROKE study [25], which is a large, active multi-centre study in the Netherlands, aiming to develop and validate an algorithm for automated signal analysis to detect anterior circulation LVO in suspected ischaemic stroke patients in the prehospital setting. The technology is fully developed; however, development of the algorithm is in early stages. The rationale for this study is underpinned by evidence that delta activity is associated with lesion location on cerebral imaging [44]. Omitting the preparation time for 'wet' EEG may enable even inexperienced EMS clinicians to undertake a measurement within $5 \mathrm{~min}$. Training requirements were not reported. Across 4 phases, algorithms will be iteratively tested and developed to maximise diagnostic accuracy using the CE-marked Waveguard $^{\mathrm{mm}}$ dry electrode cap and the eego $\mathrm{o}^{\mathrm{mm}}$ amplifier. The algorithm will be validated in the ambulance in a large multi-centre study. The EEG results will only be analysed in hospital so destination choice will not be assessed. It is not reported whether clinical outcomes will be assessed. Expected primary outcome completion was December 2019.

There are no data available on costs, EMS clinician decisions regarding hospital destination (stroke-specialist versus non-stroke specialist centre), impact on treatment or patient outcomes for either pre-hospital imaging device.

\section{Mobile telemedicine}

We identified 12 mobile telemedicine technologies reported across 15 studies [26-40]. Descriptions are summarised in Table 4. The outcomes are summarised in Table 5. Eleven studies were single-centre [26, 27, 30$33,35-38,40]$, one of which was large [30], and four were moderate-to-large multi-centre studies $[28,29,34$, 39]. From a design perspective, this included: development and pilot testing $(n=2)$, feasibility and pilot testing $(n=3)$, mixed methods pilot test $(n=1)$, retrospective before and after $(n=1)$, prospective cohort $(n=4)$, a prospective non-randomised controlled trial $(n=1)$, protocol: single-centre RCT $(n=1)$, single-centre RCT $(n=1)$.

All telemedicine systems included video and audio components, with exception of Stroke Angel in which stroke screening information was collected and 


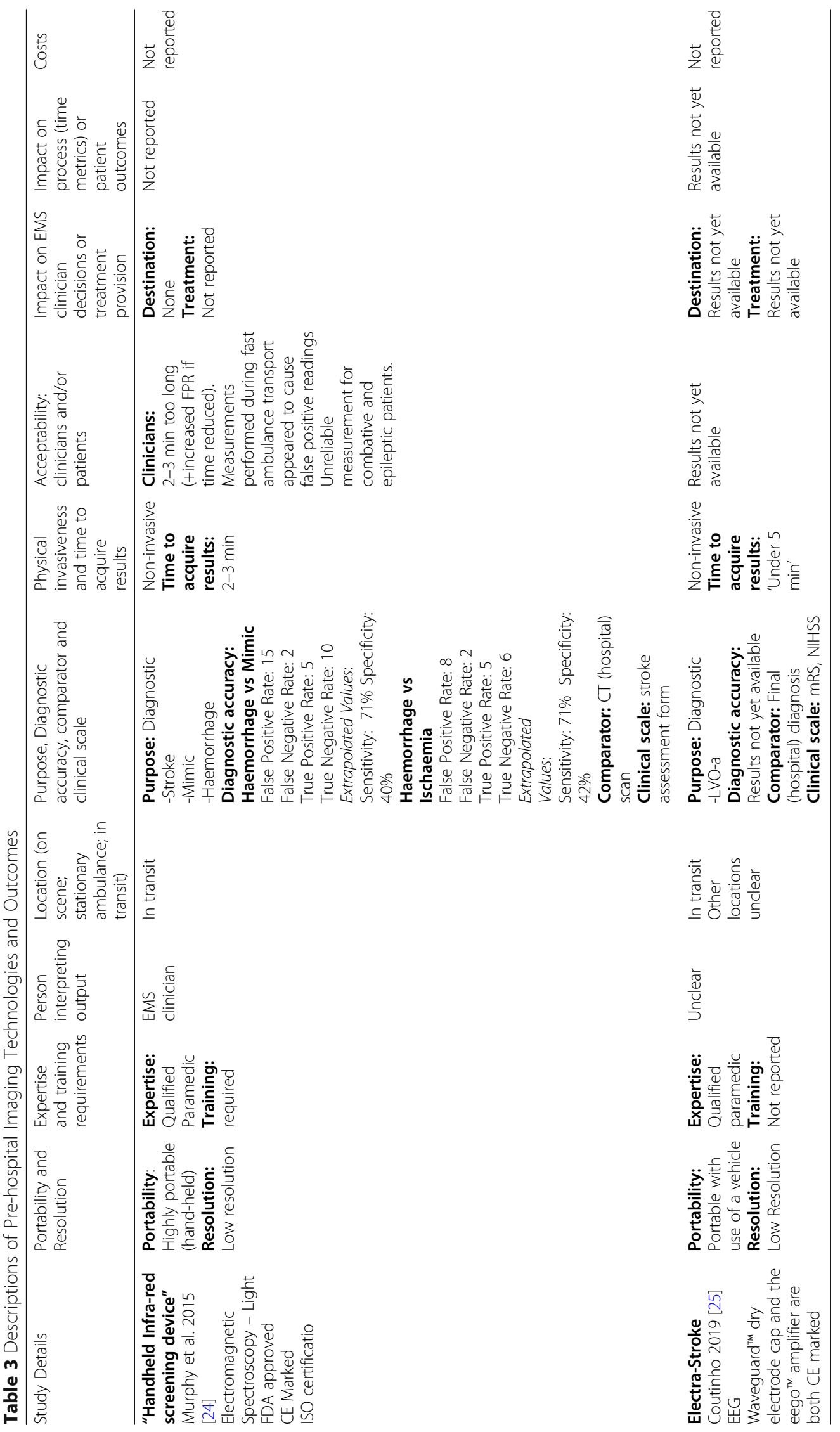




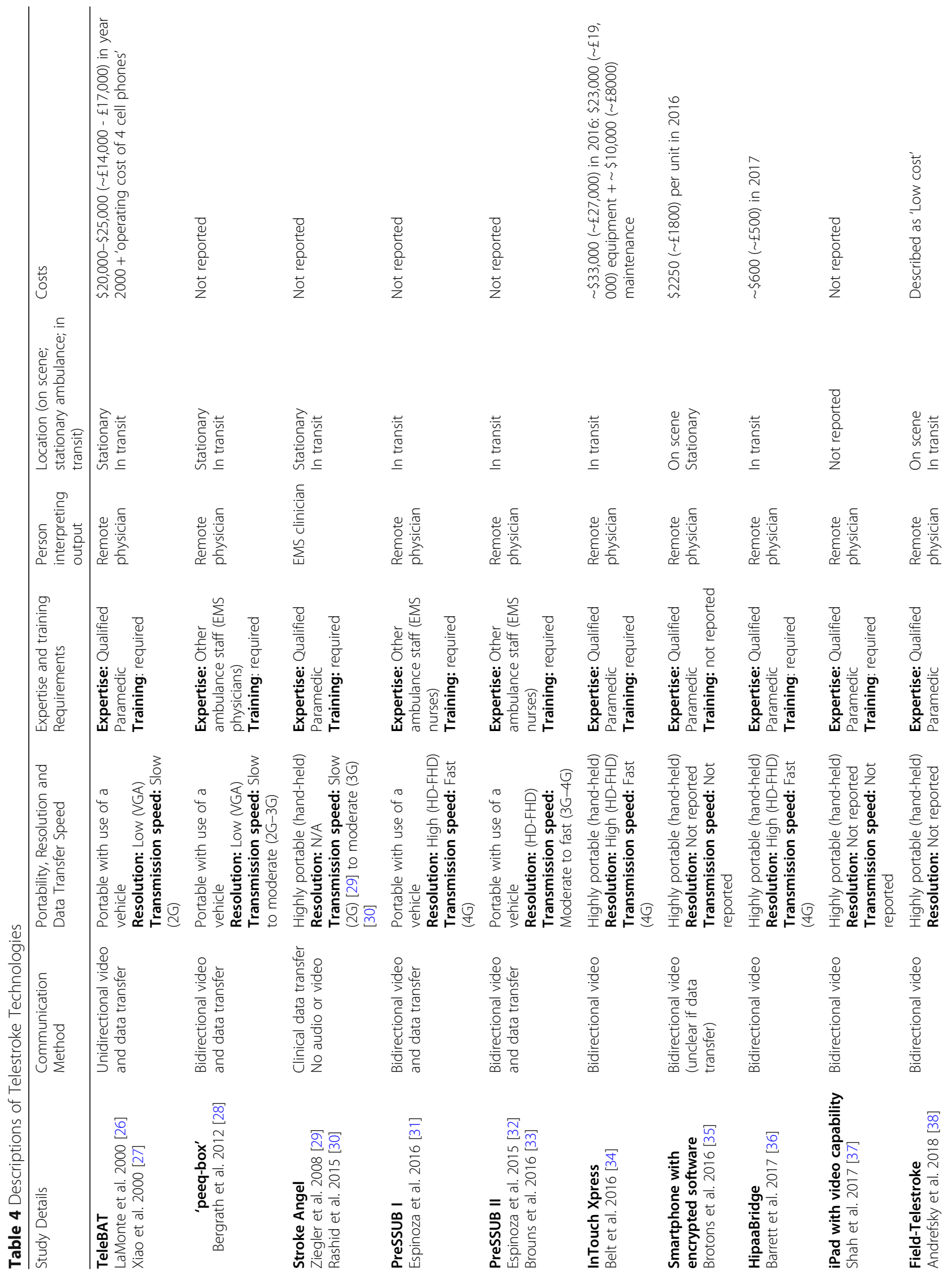




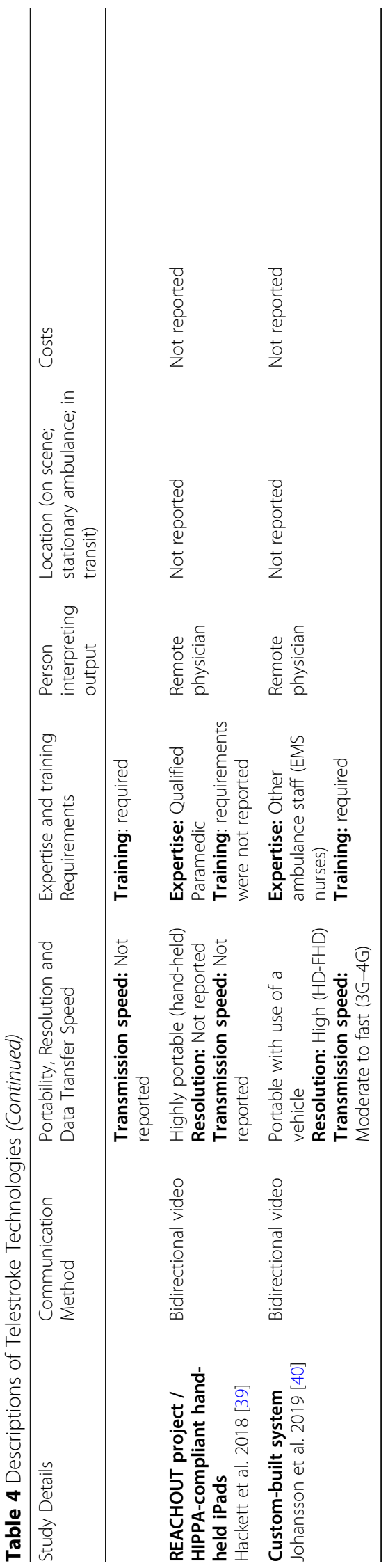




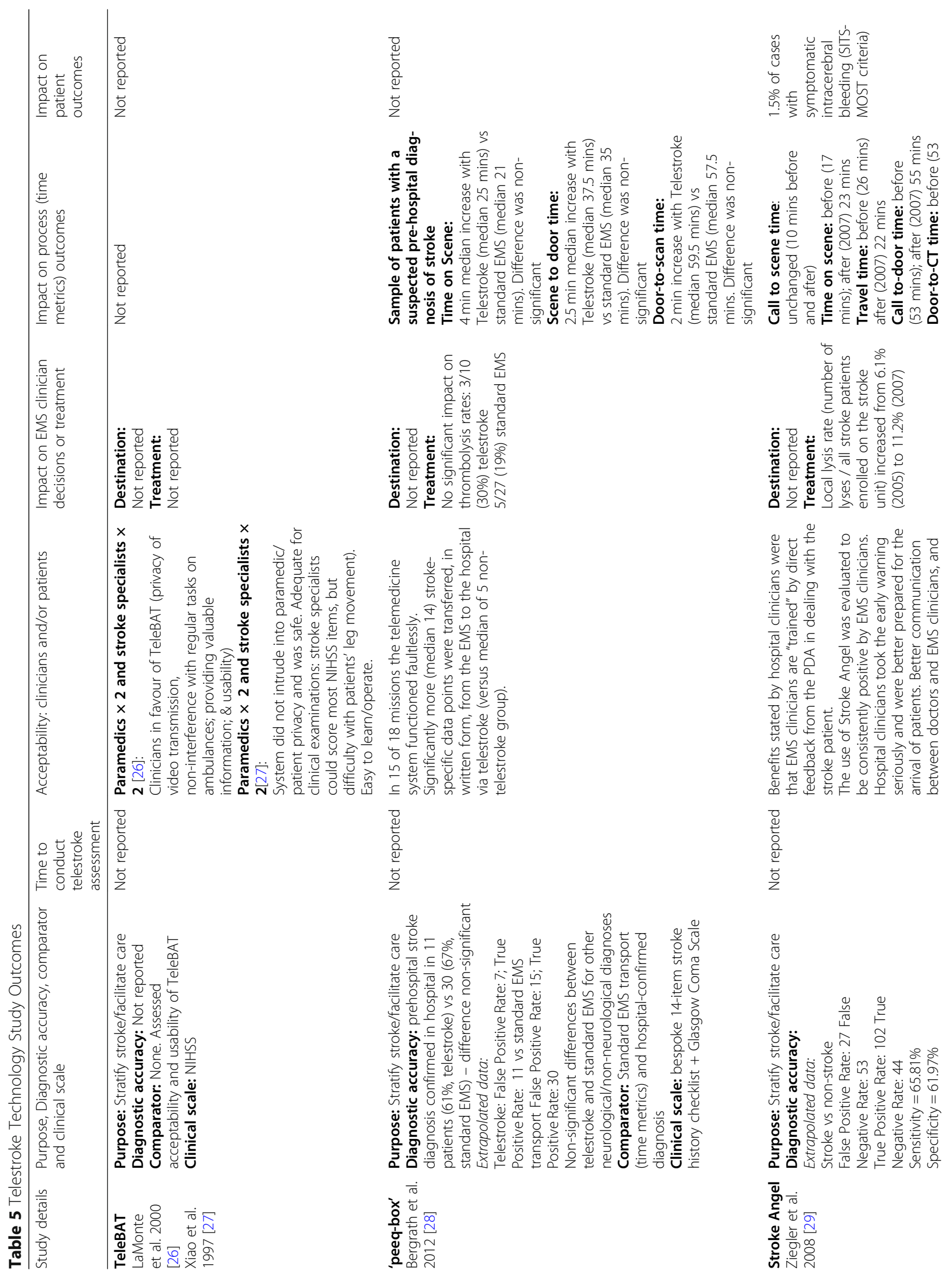




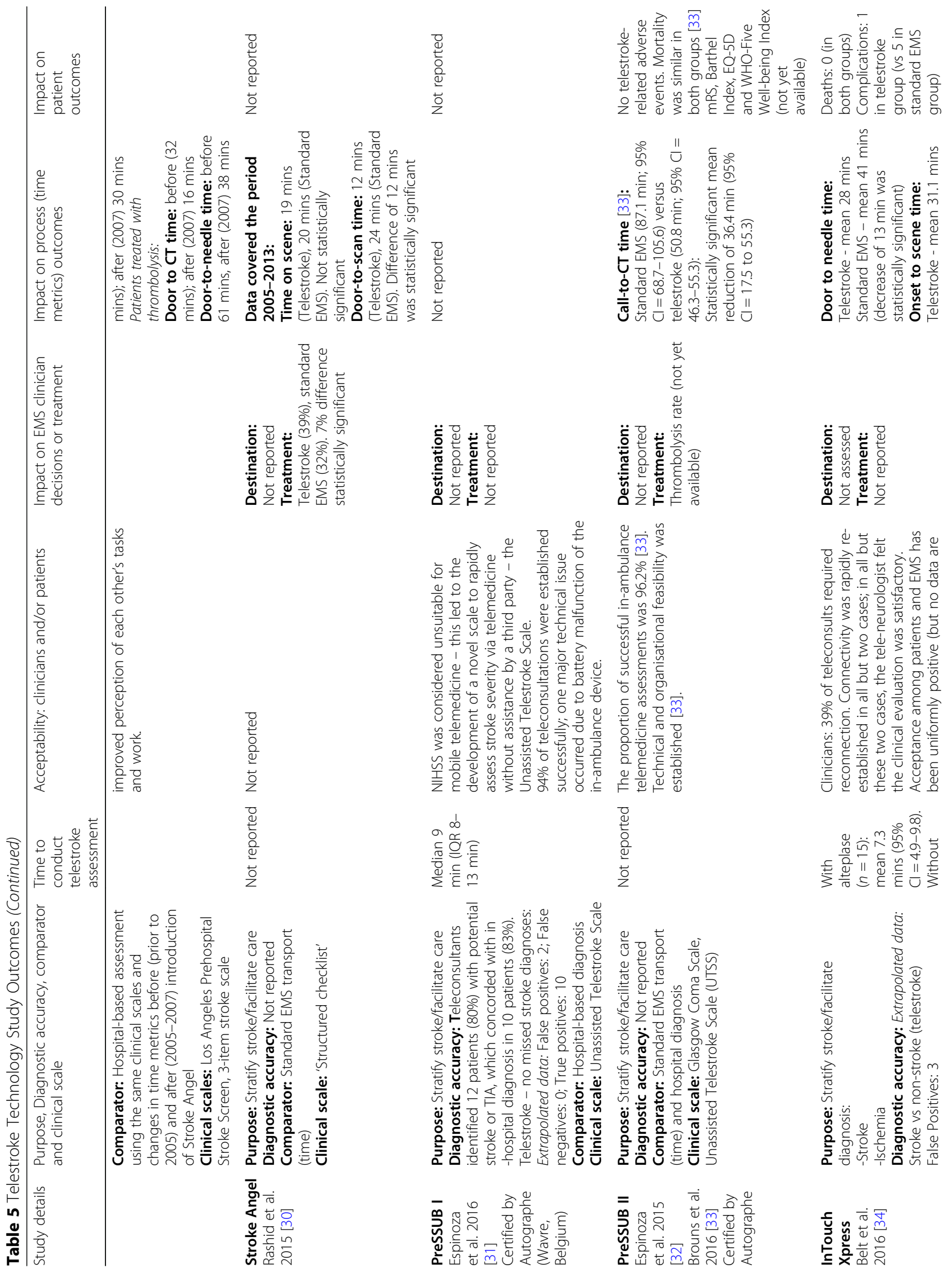




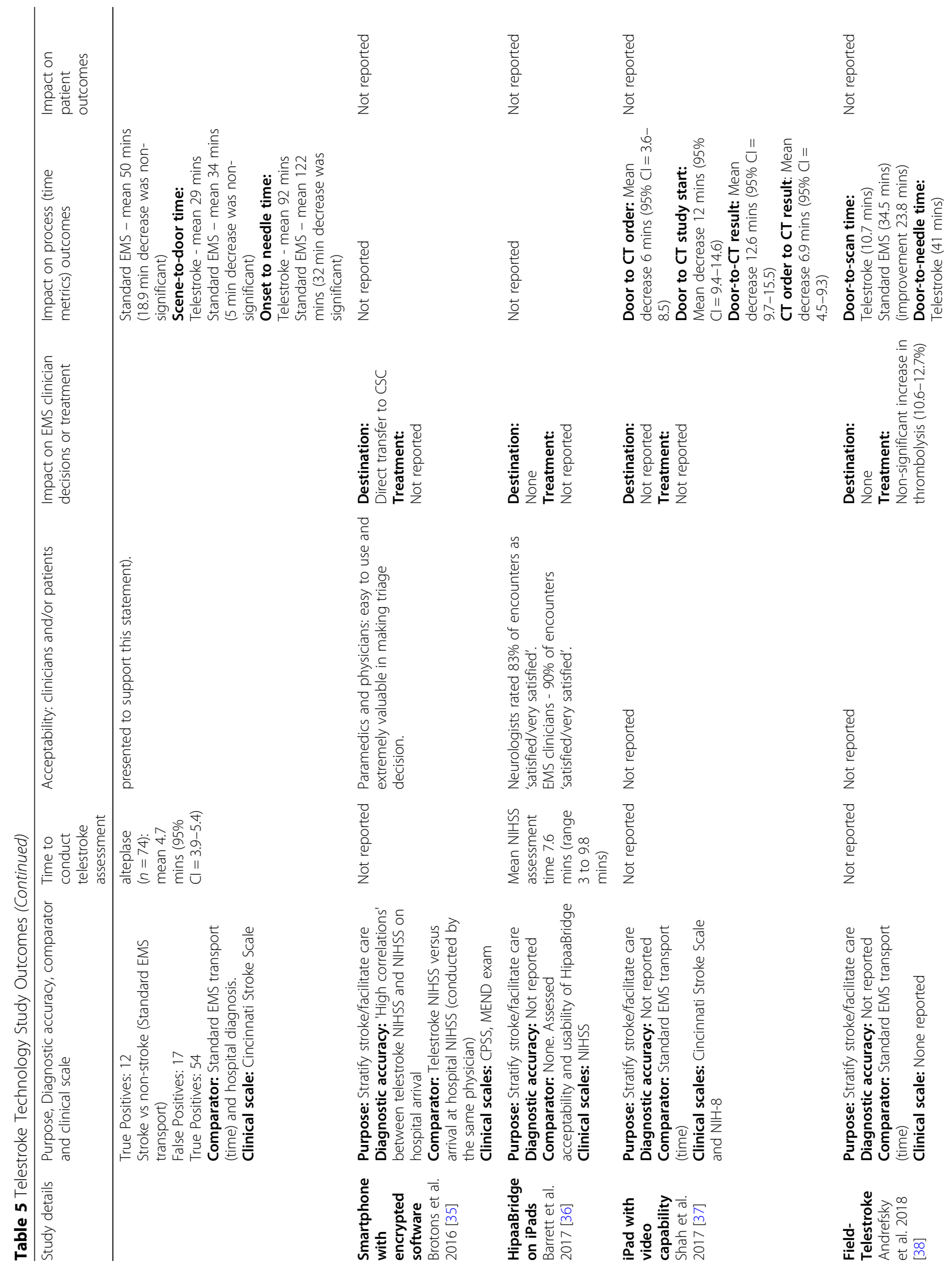




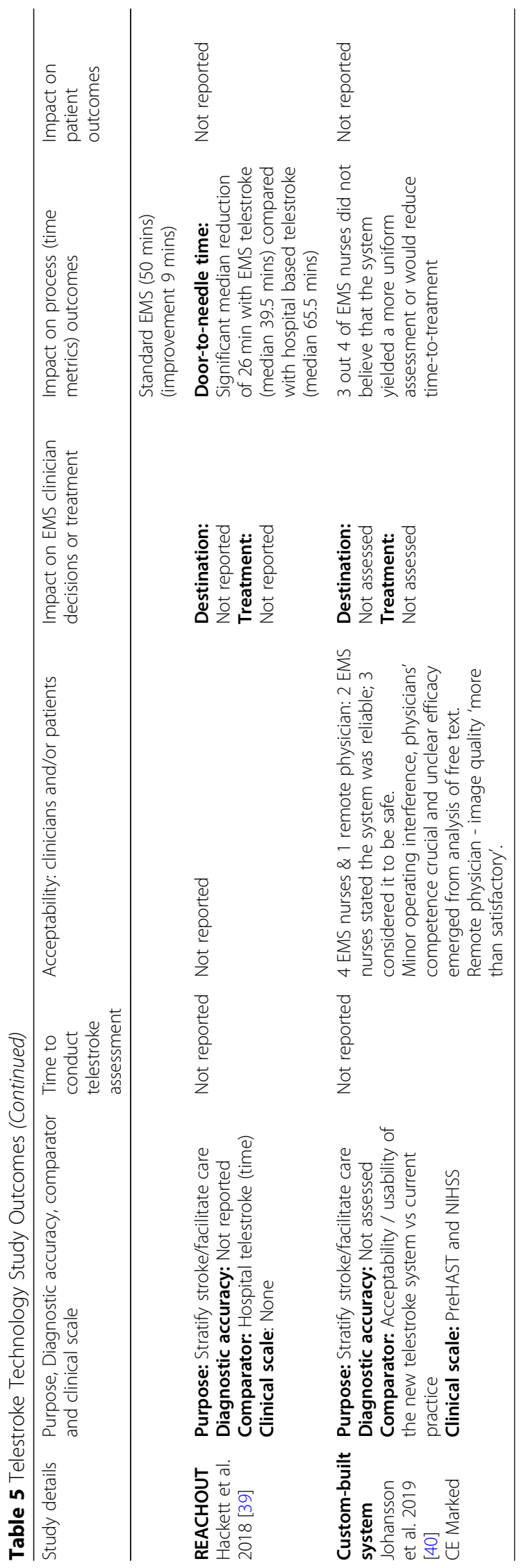


transferred from the ambulance to hospital. Earlier studies [26-30] utilised technology with lower resolution and slower transmission speeds than later studies [3140] employing contemporary technology such as high definition, bi-directional video communication and $4 \mathrm{G}$ networks. Systems were either purpose-built or adapted from commercially available technology (e.g. tablet PCs). Most telemedicine systems were in the Beta stage of development, with exception of three Gamma stage systems [30, 33, 34].

The need for EMS clinician training on use of telestroke systems was reported for all but two studies [35, 39]. EMS clinicians were predominately paramedics, with three studies employing EMS nurses (equivalent to paramedics in these countries) [31,33, 40] and one [28] EMS physicians and paramedics (with the aim of obviating the need for EMS physicians).

Costs were rarely reported, limiting comparison between studies. Where reported [26, 27, 34-36], costs are based on year of publication prices (converted costs were calculated using historical exchange rates but not adjusted for inflation). None of the studies reported on the full range of costs required to implement telestroke (training, unit, operating and maintenance).

A variety of existing and commonly used pre-hospital and hospital-based stroke screening scales were used in conjunction with the telestroke systems (Table 5). Three studies evaluated and used a bespoke telemedicine scale. A 14-item stroke history checklist was developed by experts based on published checklists and recommendations and evaluated for use in conjunction with the 'peeq-box' system [28]. The PreSSUB I and II studies $[31,32]$ developed and evaluated the Unassisted Telestroke Scale; included items were based on existing stroke scales and evaluation of their appropriateness by experts $[45,46]$.

Data on diagnostic accuracy of telestroke systems were reported in five studies [28, 29, 31, 34, 35]. Pre-hospital stroke diagnosis (versus other neurological/non-neurological diagnoses) using the 'peeq-box' telestroke system was comparable to standard EMS transport and hospital confirmed diagnoses of stroke [28]. The Stroke Angel telestroke system, utilising the Los Angeles Pre-hospital Stroke Screen, had only moderate sensitivity (66\%) and specificity (62\%) for a diagnosis of stroke in the prehospital setting [29]. The PreSSUB I study [31] reported an equivalent rate of stroke diagnosis between telestroke and hospital-based clinical assessments (80 and 83\% respectively). The InTouch Express telestroke system, using the Cincinnati Pre-hospital Stroke Scale, had equivalent rates of true/false positives for stroke diagnosis compared with standard EMS transport [34]. Finally, a smartphone telestroke system with encrypted software using the National Institute of Health Stroke Scale
(NIHSS) reported 'high' intra-rater reliability with hospital-based NIHSS assessment [35].

Eleven of 15 studies [26-29, 31-36, 40] evaluated acceptability/usability of telestroke systems from the perspective of EMS clinicians and remote physicians using mixed methods. Results were positive, with studies reporting only minor issues related to connectivity [28, $34]$ and high levels of satisfaction with systems [26, 27, $29,31,33-36]$, image quality, reliability, usability or perceived safety [26-29, 33-35, 40]. One study reported only $25 \%$ of EMS nurses believed telestroke could improve assessments and reduce time-to-treatment due to concerns about clinician ability to use systems and integration into standard care processes [40]. Robust data on patient acceptance was not reported.

Time metrics were reported for 11 of 15 telestroke studies [28-34, 36-39]. Duration of telestroke consultation was reported in three [31, 34, 36]. PreSSUB I [31] consultations were $9 \mathrm{~min}$ (IQR $8-13 \mathrm{~min}$ ). InTouch Xpress [34] consultations were 7.3 and 4.7 min (mean) for thrombolytic and non-thrombolytic patients respectively. Mean duration of NIHSS via the HipaaBridge system was $7.6 \mathrm{~min}$ [36].

With the Stroke Angel system, which allows transfer of relevant data to remote clinicians, travel time reduced by $4 \mathrm{~min}$ versus standard EMS transport [29]. Call-todoor time increased $(2 \mathrm{~min})$ and call-to-scene time matched standard care. The In-Touch Xpress study assessed onset-to-scene time [34] with a non-significant decrease of $18.9 \mathrm{~min}$. Where evaluated, there were no significant differences in time-on-scene [28-30] and scene-to-door time $[28,34]$ between telestroke and standard EMS transport. PreSSUB II was the only study to assess Call-to-CT time [33], reporting a significant mean reduction of $36.4 \mathrm{~min}(95 \% \mathrm{CI}=17.5$ to 55.3$)$ with telestroke. Door-to-CT time was improved in four studies [28-30, 37] ranging from $12 \mathrm{~min}$ [30] to $24 \mathrm{~min}$ [29]. One study utilising IPads [37] reported significantly reduced door-to-CT start $(12 \mathrm{~min})$ and result $(13 \mathrm{~min})$. Four studies reported improved door-to-needle times [29, 34, 38, 39], two of which statistically significantly [34, 39], ranging from 13 min (InTouch Xpress versus standard EMS transport) [34] to $26 \mathrm{~min}$ (REACHOUT versus hospital-based telemedicine) [39]. InTouch Xpress telestroke significantly decreased onset-to-needle time (32 min) [34].

Excluding one study, where suspected stroke patients were taken directly to the nearest specialist centre [35], telestroke studies did not assess impact on EMS clinician decisions as a function of hospital destination (strokespecialist centre versus non-specialist centre). Impact on IVT rates were assessed in four studies [28-30, 38]; two reported non-significant differences versus standard EMS transport ('peeq-box' [28] and Field-Telestroke 
[38]). Compared with standard EMS transport, the Stroke Angel system elicited significant increases in IVT, with 7\% [30] and 5\% [29] increases over 9 and 3-year periods respectively. However, neither study adjusted for concurrent increases in the thrombolysis rate. PreSSUB II [33] has yet to report on this.

Few data are available on patient safety outcomes. Stroke Angel [29] reported a 1.5\% rate of symptomatic intracerebral bleeding and $11 \%$ mortality rate with use of the system. However, a-priori rates were not reported. PreSSUB II [33] reported no telestroke-related adverse events and equivalent mortality outcomes as with standard EMS transport. The InTouch Xpress [34] telestroke system also had equivalent mortality (zero), but a lower complication rate ( 1 vs 5 respectively), compared with the standard EMS transport group. None of the telestroke studies reported on patients' functional health outcomes, although PreSSUB II [33] plans to.

\section{Discussion}

Three categories of pre-hospital technologies, with intended use by EMS clinicians to facilitate stroke care, were identified: two direct diagnosis methods (biomarkers and pre-hospital imaging) and one adjunctive technology to facilitate stratification (mobile telemedicine/telestroke). Although telemedicine systems have been in development for some years and are relatively mature, there was little robust evidence of impact on patient outcomes. Biomarker and other diagnostic technologies are at much earlier stages of development.

Blood sampling for biomarkers in the pre-hospital setting appears feasible [41]; however, there is currently no published evidence on diagnostic accuracy and patient outcomes for the studies identified: Helsinki Ultra-acute Stroke Biomarker Study [22] and PRISM [23]. The Helsinki study examines GFAP which appears promising for identifying haemorrhage at an early time point [4749]; however, this biomarker may not be robust and, as it does not identify small haemorrhages with the same performance as large ones, many not be useful to inform IVT decisions [50]. The other Helsinki biomarker, NR2 peptide, has potential for diagnosing ischaemia but data within the first $6 \mathrm{~h}$ is limited [51,52]. The role of additional biomarkers may be crucial. There is evidence supporting the measurement of purines as an indicator of cerebral ischaemia, where increases corresponded with hypo-perfusion induced by carotid clamping [53] and correlated with greater stroke volumes in the emergency department $[42,54]$. However, the applicability of this to the pre-hospital setting is currently unconfirmed. There are many other candidate stroke diagnostic biomarkers [55-65]. Inflammatory and anti-inflammatory cytokines may have utility in diagnosing ischaemia [6265], but may not be useful in the hyper-acute phase due to their late temporality after stroke onset [63-68]. Validation is challenging due to the various clinical subgroups within the suspected stroke population, wide ranges for normal values and latency of some of these biomarkers, which would also limit their application in hyper-acute diagnoses [57-61, 69]. As isolated biomarkers do not appear to have adequate accuracy for a definitive diagnosis, some evidence suggest a combination of biomarkers, reflecting various stroke-related biological processes, may be optimal [59, 70-73].

A previous review of pre-hospital imaging technologies for stroke diagnosis identified 10 devices in development [74]. However, only two devices fulfilled our review criteria of application during pre-hospital care. We identified a single-centre pre-hospital pilot study of an infrared screening device, reporting moderate sensitivity $(\sim 71 \%)$ and poor specificity $(\sim 40 \%)$ for differentiating haemorrhagic stroke from ischaemia and mimics [24] with diagnostic accuracy influenced by speed of the moving ambulance [24]. No data are available on redirection of patients to stroke-specific centres or patient health outcomes following clinical use of the device. Similar devices exist but have not been assessed in the pre-hospital setting and some not yet in humans [7578]. ELECTRA-STROKE has yet to report on outcomes. There are alternative electrophysiological devices with intended application to the pre-hospital setting [79-82] but clinical publications are lacking. Other potential prehospital imaging technologies include Volumetric Integral Phase Shift Spectroscopy (VIPS) by Cerebrotech Medical Systems, Inc., which has been evaluated in hospital but not the pre-hospital setting. VIPS uses electromagnetic induction to detect ischaemic stroke (including LVO) via hemispheric bioimpedence asymmetry. Evidence of diagnostic accuracy is promising and a new study is ongoing [83, 84]. Similar technologies exist but at an earlier stage of development without pre-hospital data reported [85-93] including magnetic particle imaging [94] ultrasonography [95-101] and accelerometery $[102,103]$.

The European Academy of Neurology and European Stroke Organization consensus statement for prehospital management of stroke did not support routine use of pre-hospital telemedicine for suspected stroke [104]. However, we identified evidence from observational studies and one RCT [26-40] of the safety, feasibility, and potential scalability of telemedicine, with equivalent diagnostic accuracy to hospital-based clinical diagnoses. Telestroke may expedite time-to-treatment by attenuating hospital-based assessment, but studies to date have shown little evidence of more efficient patient redirection to stroke-specific centres and no impact on health outcomes for specific population groups. There are no trial reports of telestroke use to improve the 
delivery of thrombectomy. Although reported running costs were relatively low, none of the studies reported on the full range of costs required for implementation (training, unit, operating and maintenance) which would inform commissioning decisions. Nevertheless, increased efficiency with telestroke (e.g., $13 \mathrm{~min}$ reductions in door-to-needle time [34]) is congruent with mobile stroke units [105], which also lack clear evidence of better patient health outcomes [104]; telestroke technology could be a significantly more cost-effective alternative in systems which do not have specialists present in the ambulance [34]. In conjunction with clinician acceptability for the majority of telestroke systems, the modest goal (stratification) permits feasibility of implementing telestroke technologies in the pre-hospital setting in the near future; however, further studies are still required. Laterstage barriers to implementation should also be addressed in prior development work and monitored with qualitative process evaluations alongside RCTs. One study evaluating the acceptability/usability of a custom-built telestroke system highlighted that EMS clinicians' views on the potential efficacy of any new system is important for successful deployment [40]. This underscores the need to engage with relevant clinicians in the development and testing of pre-hospital interventions.

In terms of quality, the included pre-hospital imaging and biomarker studies were appropriately designed to assess diagnostic accuracy, with reference standards as comparators. With exception of PRESSUB II $[32,33]$ and Stroke Angel $[29,30]$, none of the included telemedicine studies were adequately designed to formally assess diagnostic accuracy, although some confirmed the use of final clinical diagnosis as a comparator [28, 31, 35]. As telemedicine cannot provide a definitive diagnosis, it is more pertinent to assess scale reliability and reductions in timeto-treatment via triage facilitation. The majority of telemedicine studies did assess time metrics and therefore efficacy [28-30, 32, 33, 35, 37-39], but were unblinded observational reports and should be considered as service evaluations rather than definitive evidence. Apart from two telemedicine studies [32, 35], none of the studies reported or planned to report cost-effectiveness. With exception of some large studies, the majority of studies were small, single-centre studies and so lacked robust evidence.

The strengths of this review include a comprehensive structured search strategy and independent assessment at each stage of the review process. However, unpublished data reporting on the efficacy of pre-hospital stroke technology was not included. Additionally, many emerging candidate technologies at earlier research stages were omitted due to a lack of pre-hospital testing, although the intention for most is to conduct prehospital trials in the future. The results are limited by the information provided in published reports about the technology, clinical population and reference standard. There were no high quality diagnostic accuracy studies. As a decision support technology, telemedicine could act as a precursor to hospital-based imaging and so will always have limited value when used in isolation. No study combined telestroke with other technologies that may enhance stratification.

In future, efficacy of technologies aiming to provide definitive diagnoses (biomarkers and portable imaging) should be first established in the hospital setting with a clearly stated reference standard (brain imaging and specialist review) as a comparator. Using the most promising technologies, it would then be important to undertake appropriate multi-centre studies comparing standard EMS with a combination of direct stratification (portable imaging and/or biomarkers) and facilitation (telestroke) technologies, as well as additional validated pre-hospital clinical assessment scales. This would establish: 1). efficacy of stroke diagnosis and stratification across different pathways/service configurations; 2). additional value over existing pre-notification systems; 3 ). impact on service optimisation (particularly, minimisation of secondary transfer for thrombectomy); 4). whether improved process measures (e.g. time metrics and stratification) translate into clinically significant improvements in patient health and quality of life outcomes. Once validated, impact on treatment decisions and patient outcomes can be evaluated. Health economic evaluations would also provide insights into cost-effectiveness to inform decision making by commissioners.

\section{Conclusions}

Although there is clear recognition of the potential value for using emerging technology during the pre-hospital diagnosis or stratification of suspected stroke, a lack of high quality pre-hospital data on biomarkers and portable imaging technologies suggests that further development and validation in the pre-hospital setting is first required. Evaluations of telestroke systems for diagnosis and stratification of stroke indicate they are feasible and safe, but they lack robust evidence for impact on service optimisation and cost-effective patient health outcomes. Multicentre diagnostic accuracy and clinical utility studies combining these promising direct and adjunctive pre-hospital diagnostic technologies are warranted to inform recommendations on their use. Further development and validation of promising technologies has the potential to revolutionise acute stroke diagnosis and stratification.

\section{Supplementary information}

Supplementary information accompanies this paper at https://doi.org/10. 1186/s12873-020-00323-0.

Additional file 1. Search Strategy and Data Extraction Form. 


\section{Abbreviations}

MT: Mechanical Thrombectomy; LVO: Large Vessel Occlusion; FAST: Face Arm Speech Test; CT: Computed Tomography; MRI: Magnetic Resonance Imaging; MeSH: Medical Subject Headings; EMS: Emergency Medical Services;

TIDieR: Template for Intervention Description and Replication;

IVT: Intravenous Thrombolysis; mRS: modified Rankin Scale; GFAP: Glial

Fibrillary Acidic Protein; NR2: N-Methyl-D-Aspartate Receptor 2;

EEG: Electroencephalography; RCT: Randomised Controlled Trial; PC: Personal Computer; NIHSS: National Institute of Health Stroke Scale; VIPS: Volumetric Integral Phase Shift Spectroscopy

\section{Acknowledgements}

We are grateful to Alex Inskip (Newcastle University) for assistance with designing the search strategy.

\section{Author contributions}

$\mathrm{CP}$ conceived the review. CP, DF and $\mathrm{HL}$ developed the review methods. $\mathrm{HL}$ and $\mathrm{Al}$ designed and conducted the search strategy. $\mathrm{HL}$ and $\mathrm{DF}$, with assistance of CP and LS, assessed studies for inclusion. HL and DF extracted data from included studies. $H \mathrm{~L}, \mathrm{DF}$ and $\mathrm{CP}$ drafted the manuscript. $\mathrm{HL}, \mathrm{DF}$, $C P, L S, G F, P W \& G M$ were involved in the interpretation of data, critically reviewed the manuscript for intellectual content and approved the final version of the manuscript.

\section{Funding}

We did not receive any external funding to conduct this review.

\section{Availability of data and materials}

This is not applicable as no primary data were collected for this review.

\section{Ethics approval and consent to participate}

Not applicable.

\section{Consent for publication}

Not applicable.

\section{Competing interests}

$H L, D F$ and PW report no conflicts of interest. GAF, CP \& LS are investigators for two non-commercial trials of new technology to assist with acute stroke assessment (PRISM: http://www.isrctn.com/ISRCTN22323981 and ABACUS: http://www.isrctn.com/ISRCTN79169844).

\section{Author details}

${ }^{1}$ Population Health Sciences Institute, Faculty of Medical Sciences, Newcastle University, Newcastle upon Tyne, UK. ${ }^{2}$ School of Health and Social Care, Teesside University, Tees Valley, UK. ${ }^{3}$ North East Ambulance Service NHS Foundation Trust, Newcastle upon Tyne, England. ${ }^{4}$ Medical Sciences Division, Oxford Academic Health Science Network, University of Oxford, and Oxford University Hospitals NHS Foundation Trust, Oxford, UK. ${ }^{5}$ Newcastle upon Tyne Hospitals NHS Foundation Trust, Newcastle upon Tyne, England. ${ }^{6}$ Northumbria Healthcare NHS Foundation Trust, Newcastle upon Tyne, England.

Received: 12 February 2020 Accepted: 8 April 2020

Published online: 26 April 2020

\section{References}

1. Wardlaw J, Murray $\bigvee$, Berge E, del Zoppo G. Thrombolysis for acute Ischaemic stroke. Cochrane Database Syst Rev. 2014;7:CD000213.

2. National Institute for Health and Care Excellence. Alteplase for Treating Acute Ischaemic Stroke (Review of Technology Appraisal Guidance 122). 2012.

3. Saver J, Goyal M, Van der Lugt A, Menon B, Majoie C, Dippel D, et al. Time to treatment with endovascular Thrombectomy and outcomes from ischemic stroke: a meta-analysis. JAMA. 2016;316(12):1279-89.

4. Stroke Unit Trialists' Collaboration. Organised Inpatient (Stroke Unit) Care for Stroke. Cochrane Database Syst Rev. 2013;(9):CD000197.

5. Harbison J, Hossain O, Jenkinson D, Davis J, Louw S, Ford G. Diagnostic accuracy of stroke referrals from primary care, emergency room physicians, and ambulance staff using the face arm speech test. Stroke. 2003;34:71-6.
6. Rudd M, Buck D, Ford GA, Price C. A systematic review of stroke recognition instruments in hospital and pre-hospital settings. Emerg Med J. 2016;33(11): 818-22

7. Gibson L, Whiteley W. The differential diagnosis of suspected stroke: a systematic review. J Royal College Phys Edinburgh. 2013;43:114-8.

8. Ebinger M, Winter B, Wendt M, Weber JE, Waldschmidt C, Rozanski M, et al. Effect of the use of ambulance-based thrombolysis on time to thrombolysis in acute ischemic stroke: a randomized clinical trial. JAMA. 2014;311(16):1622-31.

9. Walter S, Kostopoulos P, Haass A, Keller I, Lesmeister M, Schlechtriemen T, et al. Diagnosis and treatment of patients with stroke in a Mobile stroke unit versus in hospital: a randomised controlled trial. Lancet Neurol. 2012;11(5):397-404.

10. Peter McMeekin, Philip White, Martin A James, Christopher I Price, Darren Flynn, Gary A Ford, (2017) Estimating the number of UK stroke patients eligible for endovascular thrombectomy. European Stroke Journal 2 (4):319-326.

11. Smith E, Kent D, Bulsara K, Leung L, Lichtman J, Reeves M, et al. Accuracy of prediction instruments for diagnosing large vessel occlusion in individuals with suspected stroke: a systematic review for the 2018 guidelines for the early Management of Patients with acute ischemic stroke. Stroke. 2018;49(3):e111-e22.

12. Turc G, Maïer B, Naggara $O$, Seners $P$, Isabel C, Tisserand M, et al. Clinica scales do not reliably identify acute ischemic stroke patients with largeartery occlusion. Stroke. 2016:47(6):1466-72.

13. Lin M, Sanossian N, Liebeskind D. Imaging of Prehospital stroke therapeutics. Expert Rev Cardiovasc Ther. 2015;13(9):1001-15.

14. van Gaal S, Demchuk A. Clinical and technological approaches to the Prehospital diagnosis of large vessel occlusion. Stroke. 2018;49(4):1036-43.

15. Peters M, Godfrey C, Mclnerney P, Soares C, Hanan K, Parker D. The Joanna Briggs Institute Reviewers' Manual 2015. Methodol JBI Scoping Rev. 2015.

16. Lumley H, Flynn D, Ford G, Inskip A, McClelland G, Shaw L, et al. A Review of Pre-Hospital Diagnostic Technology to Assist with Patient Stratification During the Emergency Assessment of Suspected Stroke. PROSPERO. 2018.

17. Bos N, Krol M, Veenvliet C, Plass A. Ambulance Care in Europe. Organization and Practices of Ambulance Services in 14 European Countries. Nivel. 2015.

18. Dick W. Anglo-American Vs. Franco-German emergency medical services system. Prehospital Disaster Med. 2003;18(1):29-37.

19. Hoffmann T, Glasziou P, Boutron I, Milne R, Perera R, Moher D, et al. Better reporting of interventions: template for intervention description and replication (Tidier) checklist and guide. Br Med J. 2014;348:g1687.

20. Ulloa J. Applied Biomechatronics Using Mathematical Models. Academic press. 2018

21. Carreker D The game Developer's dictionary: a multidisciplinary lexicon for professionals and students: Cengage learning; 2012.

22. Lindsberg P. Helsinki Ultra-Acute Stroke Biomarker Study. 2016. https:// clinicaltrials.gov/ct2/show/NCT02145663. 21st May 2018.

23. Shaw L, Dale N, Ford G, Graziadio S, Imray C, Lendrem C, et al Purines for Rapid Identifcation of Stroke Mimics. 2019. http://www.isrctn.com/ ISRCTN22323981. 21st May 2018.

24. Murphy D, De Kerillis P, Frabizzio J, Nash B, Shah Q. Measurement of Acute Brain Hemorrhage in the Pre-Hospital Setting. International Stroke Conference. 2015;46(SUPPL. 1).

25. Coutinho J Eeg Controlled Triage in the Ambulance for Acute Ischemic Stroke (Electra-Stroke). 2019. https://clinicaltrials.gov/ct2/show/NCT03699397. 6th June 2019.

26. LaMonte MP, Cullen J, Gagliano DM, Gunawardane R, Hu P, Mackenzie C, et al. Telebat: Mobile telemedicine for the brain attack team. J Stroke Cerebrovasc Dis. 2000;9(3):128-35.

27. Xiao Y, Gagliano D, LaMonte M, Hu P, Gaasch W, Gunawadane R, et al. Design and evaluation of a real-time Mobile telemedicine system for ambulance transport. J High Speed Networks. 2000;9(1):47-56.

28. Bergrath S, Reich A, Rossaint R, Rortgen D, Gerber J, Fischermann H, et al. Feasibility of Prehospital Teleconsultation in acute stroke - a pilot Study in clinical routine. PLoS One. 2012;7(5):e36796.

29. Ziegler V, Rashid A, Muller-Gorchs M, Kippnich U, Hiermann E, Kogerl C, et al. Mobile computing Systems in Preclinical Care of stroke: results of the stroke angel initiative within the Bmbf project Percomed. Anaesthesist. 2008;57(7):677-85

30. Rashid A, Soda H, Kippnich U, Shammas L, Hiermann E, Ziegler V, et al. Stroke angel: telemedicine Prenotification improves door-to-Ct and rate of systemic thrombolysis. Euro Stroke Org Conference. 2015;39(SUPPL. 2):33

31. Espinoza A, Van Hooff R-J, De Smedt A, Moens M, Yperzeele L, Nieboer K, et al. Development and pilot testing of 24/7 in-ambulance telemedicine for acute 
stroke: Prehospital stroke Study at the Universitair Ziekenhuis Brussel-project. Cerebrovasc Dis. 2016;42(1-2):15-22.

32. Espinoza AV, Van Hoof R-J, De Smedt A, Moens M, Yperzeele L, Nieboer K, et al. Pressub ii: the Prehospital stroke Study at the Universitair De Ziekenhuis Brussel ii. J Translational Internal Med. 2015;3(2):57-63.

33. Brouns R, Van Hooff RJ, De Smedt A, Moens M, Valenzuela Espinoza A, Lauwaert D, et al 24/7 in-Ambulance Telestroke: Results from the PreHospital Stroke Studyat the Universitair Ziekenhuis Brussel li (Pressub li). European Stroke Organisation Conference. 2016;1(1 Supplement 1):699.

34. Belt GH, Felberg RA, Rubin J, Halperin JJ. In-transit telemedicine speeds ischemic stroke treatment: preliminary results. Stroke. 2016;47(9):2413-5.

35. Brotons A, Motola I, Romano J, Schwemmer S, Issenberg B. The Use of Prehospital Telemedicine to Aid in the Decision to Airlift Patients to a Comprehensive Stroke Center from a Rural Area. International Stroke Conference. 2016;47(SUPPL. 1).

36. Barrett KM, Pizzi MA, Kesari V, TerKonda SP, Mauricio EA, Silvers SM, et al. Ambulance-based assessment of Nih stroke scale with telemedicine: a feasibility pilot Study. J Telemed Telecare. 2017;23(4):476-83.

37. Shah A, Barbera C, Bilotta M, Martin A, Sandel K, Sigal A. A Novel Use of outof-Hospital Telemedicine to Decrease Door-to-Computed Tomography Results in Acute Strokes. Research Forum. 2017;70(4 Supplement 1):S117.

38. Andrefsky J, Rose S, Chase N, Ellenberger D, Fussner JM, Sila C. Impact of Ems Field-Telestroke with Hand-Held Ipads on Iv-Tpa Therapy for Stroke. International Stroke Conference and State-of-the-Science Stroke Nursing Symposium. 2018;49(Supplement 1).

39. Hackett CT, Protetch J, Rana SS, Wright DG, Fishman R, Schmidt EC, et al Ems Based Telestroke Suggests Reduced Door to Needle Time Compared to Hospital Based Telestroke (Reachout Project). International Stroke Conference and State-of-the-Science Stroke Nursing Symposium. 2018;49(Supplement 1).

40. Johansson A, Esbjornsson M, Nordqvist P, Wiinberg S, Andersson R, Ivarsson $B$, et al. Technical feasibility and ambulance Nurses' view of a digital telemedicine system in pre-hospital stroke care - a pilot Study. Int Emerg Nurs. 2019;44:35-40.

41. Mattila $\mathrm{O}$, Harve $\mathrm{H}$, Pihlasviita $\mathrm{S}$, et al. Ultra-acute diagnostics for stroke: large-scale implementation of Prehospital biomarker sampling. Acta Neurol Scand. 2017;136(1):17-23.

42. Dale N, Tian F, Imray C, Roffe C. Purines are rapidly released into venous blood following stroke. UK Stroke Forum. 2015;10:16.

43. NIHR Horizon Scanning Research \& Intelligence Centre. Smartchip for early diagnosis of suspected stroke. 2016. http://www.io.nihr.ac.uk/wp-content/ uploads/migrated/SMARTChip-Horizon-Scanning-alert-FINAL.pdf.

44. Finnigan S, Van Putten M, et al. EEG in Ischaemic stroke: quantitative EEG can uniquely inform (sub-)acute prognoses and clinical management. Clin Neurophysiol. 2013;124(1):10-9.

45. Van Hooff R, De Smedt A, De Raedt S, Moens M, Mariën P, Paquier P, et al. Unassisted assessment of stroke severity using telemedicine. Stroke. 2013; 44(5):1249-55

46. Van Hooff R, Cambron M, Van Dyck R, De Smedt A, Moens M, Espinoza A, et al. Prehospital unassisted assessment of stroke severity using telemedicine: a feasibility Study. Stroke. 2013;44(10):2907-9.

47. Foerch C, Pfeilschifter W, Zeiner P, Brunkhorst R. Acidic glia Fiber protein in patients with acute stroke symptoms. Neurologist. 2014;85(8):982-9.

48. Luger S, Witsch J, Dietz A, Hamann G, Minnerup J, Schneider H, et al. Glial Fibrillary acidic protein serum levels distinguish between Intracerebral hemorrhage and cerebral ischemia in the early phase of stroke. Clin Chem. 2017;63(1):377-85.

49. Garcia-Berrocoso T, Llombart V, Giralt D, Bustamante A, Penalba A, Boada C, et al Brain-Specific Proteins NEF3, CARNS1 and B-Synuclein Combined with GFAP as Biomarkers for Stroke Subtype Differentiation. European Stroke Journal. 2016; European Stroke Organisation Conference.

50. Rozanski M, Waldschmidt C, Kunz A, Grittner U, Ebinger M, Wendt M, et al. Glial Fibrillary acidic protein for Prehospital diagnosis of Intracerebral hemorrhage. Cerebrovasc Dis. 2017;43(1-2):76-81.

51. Dambinova S, Bettermann K, Glynn T, Tews M, Olson D, Weissman J, et al. Diagnostic potential of the NMDA receptor peptide assay for acute ischemic stroke. PLoS One. 2012;7(7):e42362.

52. Dambinova S. Is Nr2 peptide promising biomarker for acute cerebrovascular events? Stroke. 2013;44:A30.

53. Tian F, Bibi F, Dale N, Imray C. Blood purine measurements as a rapid realtime Indicator of reversible brain Ischaemia. Purinergic Signalling. 2017;13(4): 521-8.
54. Dale N, Tian F, Sagoo R, Phillips N, Imray C, C R. Point-of-care measurements reveal release of purines into venous blood of stroke patients. Purinergic Signalling. 2019;15(2):237-46.

55. Dai D, Thajeb P, Tu C, Chiang F, Chen C, Yang R, et al. Plasma concentration of Scube1, a novel platelet protein, is elevated in patients with acute coronary syndrome and ischemic stroke. J Am Coll Cardiol. 2008;51(22):2173-80.

56. Bustamante A, López-Cancio E, Pich S, Penalba A, Giralt D, García-Berrocoso T, et al. Blood biomarkers for the early diagnosis of stroke: the stroke-Chip Study. Stroke. 2017;48(9):2419-25.

57. Misra S, Kumar A, Kumar P, Yadav A, Mohania D, Pandit A, et al. Bloodbased protein biomarkers for stroke differentiation: a systematic review. PROTEOMICS-Clin Appl. 2017;11(9-10):1700007.

58. Jickling G, Sharp F. Blood biomarkers of ischemic stroke. Neurotherapeutics. 2011;8(3):349.

59. Saenger A, Christenson R. Stroke biomarkers: Progress and challenges for diagnosis, prognosis, differentiation, and treatment. Clin Chem. 2010;56(1): 21-33.

60. Maestrini I, Ducroquet A, Moulin S, Leys D, Cordonnier C, Bordet R. Blood biomarkers in the early stage of cerebral ischemia. Rev Neurol. 2016;172(3): 198-219.

61. Whiteley $W$, Tseng M, Sandercock P. Blood biomarkers in the diagnosis of ischemic stroke: a systematic review. Stroke. 2008;39(10):2902-9.

62. Tuttolomondo A, Di Raimondo D, di Sciacca R, Pinto A, Licata G. Inflammatory cytokines in acute ischemic stroke. Curr Pharm Des. 2008; 14(33):3574-89.

63. Basic V, Simundic A, Nikolac N, Demarin V. Pro-inflammatory and antiinflammatory cytokines in acute ischemic stroke and their relation to early neurological deficit and stroke outcome. Clin Biochem. 2008;41(16-17): 1330-4.

64. Ramiro L, Simats A, García-Berrocoso T, Montaner J. Inflammatory molecules might become both biomarkers and therapeutic targets for stroke management. Ther Adv Neurol Disord. 2018;11:1756286418789340.

65. Waje-Andreassen U, Kråkenes J, Ulvestad E, Thomassen L, Myhr K, Aarseth J, et al. II-6: an early marker for outcome in acute ischemic stroke. Acta Neurol Scand. 2005;111(6):360-5.

66. Perini F, Morra M, Alecci M, Galloni E, Marchi M, Toso V. Temporal profile of serum anti-inflammatory and pro-inflammatory interleukins in acute ischemic stroke patients. Neurol Sci. 2001;22(4):289-96.

67. Mazzotta G, Sarchielli P, Caso V, Paciaroni M, Floridi A, Floridi A, et al. Different cytokine levels in thrombolysis patients as predictors for clinical outcome. Eur J Neurol. 2004;11(6):377-81.

68. Nayak A, Kashyap R, Kabra D, Purohit H, Taori G, Daginawala H. Time course of inflammatory cytokines in acute ischemic stroke patients and their relation to inter-Alfa trypsin inhibitor heavy chain 4 and outcome. Ann Indian Acad Neurol. 2012;15(3):181.

69. Maas M, Furie K. Molecular biomarkers in stroke diagnosis and prognosis. Biomark Med. 2009:3(4):363-83.

70. Sharma R, Macy S, Richardson K, Lokhnygina Y, Laskowitz D. The Development and Validation of a Biochemical Biomarker Panel to Detect Acute Stroke: A Preliminary Study. Stroke. 2013;44(Supplement 1):A31.

71. Sharma R, Macy S, Richardson K, Lokhnygina Y, Laskowitz D. A blood-based biomarker panel to detect acute stroke. J Stroke Cerebrovasc Dis. 2014;23(5): 910-8.

72. Penn A, Saly V, Trivedi A, Lesperance M, Votova K, Jackson A, et al. Differential Proteomics for distinguishing ischemic stroke from controls: a pilot Study of the spectra project. Transl Stroke Res. 2018;9(6):590-9.

73. Llombart V, García-Berrocoso T, Bustamante A, Giralt D, Rodriguez-Luna D, Muchada $\mathrm{M}$, et al. Plasmatic retinol-binding protein 4 and glial Fibrillary acidic protein as biomarkers to differentiate ischemic stroke and Intracerebral hemorrhage. J Neurochem. 2016;136(2):416-24.

74. Walsh K. Non-invasive sensor Technology for Prehospital Stroke Diagnosis: current status and future directions. Int J Stroke. 2019;14(6):592-602.

75. Moreau F, Yang R, Nambiar V, Demchuk A, Dunn J. Near-infrared measurements of brain oxygenation in stroke. Neurophotonics. 2016;3(3): 031403.

76. Chang G, Wang K, Hsu C, Chen J. Development of functional near infrared spectroscopy system for assessing cerebral hemodynamics of rats with ischemic stroke. J Med Biol Eng. 2007;27(4):207.

77. Robertson C, Zager E, Narayan R, Handly N, Sharma A, Hanley D, et al. Clinical evaluation of a portable near-infrared device for detection of traumatic intracranial hematomas. J Neurotrauma. 2010;27(9):1597-604. 
78. Infrascanner.com. Portable Brain Scanner: Head Injury Assessment Tool | Infrascan. [online]. 2020. Available at: [Accessed 14 April 2020].

79. Forestdevices.com. Forest Devices - Stroke Decisions Made Smarter. [online]. 2020. Available at: [Accessed 14 April 2020].

80. Michelson E, Hanley D, Chabot R, Prichep L. Identification of acute stroke using quantified brain electrical activity. Acad Emerg Med. 2015;22(1):67-72.

81. Bonmassar G, Iwaki S, Goldmakher G, Angelone L, Belliveau J, Lev M. On the measurement of electrical impedance spectroscopy (Eis) of the human head. Int J Bioelectromagnetism. 2010;12(1):32.

82. Xu C, Wang L, Shi X, You F, Fu F, Liu R, et al. Real-time imaging and detection of intracranial Haemorrhage by electrical impedance tomography in a piglet model. J Int Med Res. 2010;38(5):1596-604.

83. Kellner C, Sauvageau E, Snyder K, Fargen K, Arthur A, Turner R, et al. The vital Study and overall pooled analysis with the Vips non-invasive stroke detection device. J Neurointerventional Surgery. 2018;10(11):1079-84.

84. Sutcliffe L. Evaluation of a new portable test to identify patients with complex stroke. ISRCTN Registry. 2018.

85. Persson M, Fhager A, Trefná H, Yu Y, McKelvey T, Pegenius G, et al. Microwave-based stroke diagnosis making global Prehospital thrombolytic treatment possible. IEEE Trans Biomed Eng. 2014;61(11):2806-17.

86. Bisio I, Estatico C, Fedeli A, Lavagetto F, Pastorino M, Randazzo A, et al. Brain stroke microwave imaging by means of a Newton-conjugate-gradient method in L(p) Banach spaces. IEEE Transactions Microwave Theory Techniques. 2018;66(8):3668-82.

87. Scapaticci R, Tobon J, Bellizzi G, Vipiana F, Crocco L. Design and numerical characterization of a low-complexity microwave device for brain stroke monitoring. IEEE Trans Antennas Propag. 2018;66(12):7328-38.

88. Merunka I, Massa A, Vrba D, Fiser O, Salucci M, Vrba J. Microwave Tomography System for Methodical Testing of Human Brain Stroke Detection Approaches. International Journal of Antennas and Propagation. 2019.

89. Yan Q, Jin G, Ma K, Qin M, Zhuang W, Sun J. Magnetic inductive phase shift: a new method to Diferentiate hemorrhagic stroke from ischemic stroke on rabbit. Biomed Eng Online. 2017;16:63.

90. Hopfer M, Planas R, Hamidipour A, Henriksson T, Semenov S. Electromagnetic tomography for detection, differentiation, and monitoring of brain stroke: a virtual data and human head phantom Study. IEEE Antennas Propagation Magazine. 2017:59(5):86-97.

91. Kandadai M, Korfhagen J, Beiler S, Beiler C, Wagner K, Adeoye O, et al. In vivo testing of a non-invasive prototype device for the continuous monitoring of Intracerebral hemorrhage. J Neurosci Methods. 2014;235:11722.

92. Poltschak S, Freilinger M, Feger R, Stelzer A, Hamidipour A, Henriksson T, et al. A multiport vector network analyzer with high-precision and Realtime capabilities for brain imaging and stroke detection. Int J Microw Wirel Technol. 2018;10(5-6):605-12.

93. Guo L, Abbosh A. Stroke localization and classification using microwave tomography with K-means clustering and support vector machine. Bioelectromagnetics. 2018;39(4):312-24.

94. Ludewig P, Gdaniec N, Sedlacik J, Forkert N, Szwargulski P, Graeser M, et al. Magnetic particle imaging for real-time perfusion imaging in acute stroke. ACS Nano. 2017;11(10):10480-8.

95. Thorpe SG, Thibeault CM, Canac N, Wilk SJ, Devlin T, RB H Decision Criteria for Large Vessel Occlusion Using Transcranial Doppler Waveform Morphology Frontiers in neurology 2018;9:847.

96. Demchuk A, Christou I, Wein T, Felberg R, Malkoff M, Grotta J, et al. Specific Transcranial Doppler flow findings related to the presence and site of arterial occlusion. Stroke. 2000;31(1):140-6.

97. Seidel G, Kaps M, Gerriets T. Potential and limitations of Transcranial colorcoded Sonography in stroke patients. Stroke. 1995;26(11):2061-6.

98. Mort A, Eadie L, Regan L, Macaden A, Heaney D, Bouamrane M, et al. Combining Transcranial ultrasound with intelligent communication methods to enhance the remote assessment and Management of Stroke Patients: framework for a technology demonstrator. Health Informatics J. 2016;22(3):691-701.

99. Eadie L, Mulhern J, Regan L, Mort A, Shannon H, Macaden A, et al. Remotely supported Prehospital ultrasound: a feasibility Study of real-time image transmission and expert guidance to aid diagnosis in remote and rural communities. J Telemed Telecare. 2018;24(9):616-22.

100. Thorpe S, Thibeault C, O'Brien M, Ranjbaran M, Canac N, Martinez-Torres L, et al Comparison of Human and Robotically Acquired Transcranial Doppler
Waveforms Towards Automated Assessment of Acute Ischemic Stroke. European Journal of Neurology Special Issue: 23rd Meeting of the ESNCH: Book of Abstracts, Prague, Czech Republic, April 13-16, 2018. 2018;25(S1): P28.

101. Thorpe S, Thibeault C, Canac N, O'Brien M, Ranjbaran M, Martinez-Torres L, et al A Novel Metric for Transcranial Doppler Assessment of Acute Ischemic Stroke. European Journal of Neurology Special Issue: 23rd Meeting of the ESNCH: Book of Abstracts, Prague, Czech Republic, April 13-16, 2018. 2018; 25(S1):055.

102. Smith W, Browne J, Ko N. Cranial Accelerometry can detect cerebral vasospasm caused by subarachnoid hemorrhage. Neurocrit Care. 2015;23(3): 364-9.

103. Smith W. Head pulse for ischemic stroke detection (episode). ClinicalTrialsgov. 2019.

104. Kobayashi A, Czlonkowska A, Ford G, Fonseca A, Luijckx G, Korv J, et al. European academy of neurology and European stroke organization consensus statement and practical guidance for pre-hospital management of stroke. Eur J Neurol. 2018;25(3):425-33.

105. Matthias Wendt, Martin Ebinger, Alexander Kunz, Michal Rozanski, Carolin Waldschmidt, Joachim E. Weber, Benjamin Winter, Peter M. Koch, Erik Freitag, Jenrik Reich, Daniel Schremmer, Heinrich J. Audebert, (2015) Improved Prehospital Triage of Patients With Stroke in a Specialized Stroke Ambulance. Stroke 46 (3):740-745

\section{Publisher's Note}

Springer Nature remains neutral with regard to jurisdictional claims in published maps and institutional affiliations.

\section{Ready to submit your research? Choose BMC and benefit from:}

- fast, convenient online submission

- thorough peer review by experienced researchers in your field

- rapid publication on acceptance

- support for research data, including large and complex data types

- gold Open Access which fosters wider collaboration and increased citations

- maximum visibility for your research: over $100 \mathrm{M}$ website views per year

At $\mathrm{BMC}$, research is always in progress.

Learn more biomedcentral.com/submissions 